\title{
Manifestaciones psíquicas y somáticas de ansiedad en adolescentes de secundaria
}

\author{
De Avila-Arroyo, María Luz*; López-Méndez, Edna Edith²;Tenahua-Quitl, Inés³; \\ Gallegos-Acevedo, Ma. Guadalupe4; Torres-Reyes, Alejandro ${ }^{5}$
}

\begin{abstract}
RESUMEN
Introducción: Las alteraciones mentales entre la población se han convertido en un problema de salud, en los adolescentes destaca la presencia de ansiedad, esta se presenta como un estado patológico que puede ocasionar disfunción física, psicológica y conductual afectando diferentes áreas de la vida. Objetivo: Identificar las manifestaciones psíquicas y somáticas de la ansiedad en adolescentes de secundaria; determinar la intensidad de la ansiedad y las diferencias por edad y sexo, e identificar la relación de la ansiedad con la edad. Metodología: Estudio correlacional, se llevó a cabo en una muestra de 312 adolescentes estudiantes de secundaria del estado de Puebla de 11 a 16 años, se realizó un muestreo aleatorio estratificado por grado y grupo escolar, se utilizó la Escala de Ansiedad de Hamilton, el estudio se apegó a la Ley General de Salud, se protegió la privacidad y confidencialidad de la información. Para el análisis estadístico se aplicó la Prueba de Chi Cuadrada y correlación de Spearman. Resultados: La mayoría de los adolescentes manifiestan síntomas psíquicos como dificultad en la concentración (57.5\%) y somáticos sensaciones de calor y frío (52.8\%) en el último mes y siete días; más del $40 \%$ de adolescentes presentan ansiedad moderada/severa, no se encontró diferencia estadísticamente significativa por edad y sexo, ni relación entre la ansiedad y edad ( $p>.05)$. Conclusiones: Los adolescentes mantienen manifestaciones psíquicas y somáticas de ansiedad desde edades tempranas que pueden generar alteraciones en diferentes áreas y etapas de la vida, es necesario realizar intervenciones de enfermería para prevenir complicaciones.
\end{abstract}

Palabras clave: Ansiedad; Síntomas psíquicos; Síntomas sin Explicación Médica; Síntomas Somáticos (DeCS;BIREME).

\footnotetext{
${ }^{1}$ Maestra en Ciencias de Enfermería. Benemérita Universidad Autónoma de Puebla, Facultad de Enfermería. México. E-mail: maluz_deavila@hotmail.com

${ }^{2}$ Maestra en Enfermería. Instituto de Seguridad y Servicios Sociales de los Trabajadores del Estado, Hospital Regional Puebla. México. E-mail: lic.edna@hotmail.com

${ }^{3}$ Maestra en Ciencias de Enfermería. Benemérita Universidad Autónoma de Puebla, Facultad de Enfermería. México. E-mail: inesteq@ gmail.com

${ }^{4}$ Maestra en Ciencias de Enfermería. Universidad Autónoma de Zacatecas, Unidad Académica de Enfermería. México. E-mail: marialupitagacevedo@yahooo.com.mx

${ }^{5}$ Maestro en Enfermería. Benemérita Universidad Autónoma de Puebla, Facultad de Enfermería. México. E-mail: alexanderreyes20102029@gmail.com
}

*Autor para correspondencia

\section{Cómo citar este artículo}

De Avila-Arroyo ML, López-Méndez EE, Tenahua-Quitl I, Gallegos-Acevedo MG. Manifestaciones psíquicas y somáticas de ansiedad en adolescentes de secundarial. Sanus. 2018;3(7): 8-23. [Acceso ]; Disponible en: mes día año 


\section{INTRODUCCIÓN}

Las alteraciones mentales entre la población se han convertido en un problema de salud, en los adolescentes destaca la presencia de ansiedad que es una emoción que forma parte de la supervivencia ya que es una respuesta ante situaciones sorpresivas o amenazantes que sirve como defensa para alertar ante un peligro. Estas reacciones son fisiológicas cuando se estimulan ante la presencia de riesgo inmediato para resguardar la integridad de una persona, significa que hay una adaptación ante las circunstancias que se exhibieron, es decir una función normal de un sistema del organismo que cuando existe un estímulo de desadaptación y se muestra en el momento que no haya una circunstancia que lo justifique, existirá un incremento en la intensidad y frecuencia del estímulo, esto se convierte en un estado emocional en el que la persona no es capaz de sentirse calmada, manifiesta irritabilidad, preocupación o alteraciones fisiológicas y de la conducta, lo que indica a la ansiedad como un estado patológico o trastorno que puede ocasionar disfunción física, psicológica y conductual afectando diferentes áreas de la vida ${ }^{(1-3)}$.

Por otro lado, es importante señalar que la ansiedad también puede exteriorizar como un síntoma de enfermedades médicas, psiquiátricas o como una entidad específica de la patología mental (2), en la que existen factores biológicos, psicológicos y sociales que predisponen al individuo a que la padezca (1). Este panorama muestra diversas causas que pueden explicar el desarrollo de la ansiedad, de tal manera que es importante estudiar esta variable.

Algunos estudios reportan como síntomas somáticos a aquellos que surgen cuando hay ansiedad, mareos, sensación de cabeza vacía, sudoración, palpitaciones, sincope, parestesia y molestias digestivas que se consideran como manifestaciones corporales que significan alteraciones físicas de órganos y sistemas ${ }^{(2-5)}$. También existen síntomas cognoscitivos, conductuales y afectivos denominados psíquicos, entre ellos destacan la inquietud, nerviosismo, miedos, insomnio y alteraciones en la función intelectual ${ }^{(1-4,6-8)}$ que se manifiestan cuando se inhiben sustancias cerebrales como la serotonina y dopamina, esto sucede cuando hay problemas en alguna área del contexto biopsicosocial del individuo. En consecuencia se ostentan alteraciones en el comportamiento y estado de ánimo, lo que reflejan una vertiente motriz-neurovegetativa, cognoscitiva, de percepción de la realidad, emocional y afectiva ${ }^{(1,2)}$.

Es importante señalar que en México la edad promedio en la cual inician los trastornos de ansiedad es a los 15 años y adolescentes de 12 a 15 tienen ansiedad, estos datos son importantes ya que durante la etapa de la adolescencia existen diversos cambios físicos y conductuales que se generan en los individuos y los hacen vulnerables a que las manifestaciones de la ansiedad se agudicen y surjan problemas ${ }^{(1,11-12)}$. Por otro lado, se documenta que a partir de esta etapa la ansiedad afecta por igual a hombres y mujeres aunque su incidencia no es clara $^{(2)}$, un estudio en adolescentes mexicanos indica que no encontró diferencias por sexo ${ }^{(11)}$, sin embargo, hay literatura de España y México que revelan que se presenta más en mujeres que en hombres ${ }^{(9,12)}$.

Por otro lado, la evidencia científica ha reportado porcentajes de trastorno de ansiedad entre los adolescentes españoles por encima de los parámetros establecidos (26.41\%), se destaca que síntomas relacionados con la ansiedad por separación y los miedos físicos son los que más se exteriorizan ${ }^{(9)}$. Además, otros resultados indican que los síntomas psicosomáticos que tuvieron mayor incidencia en estudiantes de secundaria fueron la dificultad para concentrarse y dormir, dolor de cabeza y estómago, también sentirse tenso, triste, y que los estudiantes adolescentes de más edad tuvieron las puntuaciones mayores de ansiedad con promedios de 7.4 superiores a sus puntos de corte ${ }^{(9,13)}$.

Cabe destacar que, pese a la búsqueda de literatura de la ansiedad en adolescentes, esta ha sido limitada además los reportes encontrados tienen diversas formas de medición de la variable. Lo que sugiere la necesidad indagar más sobre este tema, debido a que se reporta como un problema de salud mental que inicia durante la infancia o adolescencia y puede continuar hasta la adultez ${ }^{(1)}$, también generar alteraciones en la vida de los individuos y altos costos en la salud pública, al ser un factor de riesgo para el consumo de drogas, la perdida de la identidad, suicidio y alteraciones familiares lo que desencadenaría mayores problemas de salud ${ }^{(14)}$. De tal manera que se hace necesario diseñar estrategias de salud para prevenir, detectar y manejar esta problemática con la finalidad de evitar complicaciones futuras esto puede ser a través de intervenciones de enfermería específicas para la atención de adolescentes con ansiedad.

Por lo tanto surgen los objetivos de identificar las manifestaciones psíquicas y somáticas de la ansiedad en adolescentes de secundaria del estado de Puebla, asimismo determinar la intensidad de la ansiedad y las diferencias por edad y sexo e identificar la relación de la ansiedad con la edad.

\section{METODOLOGÍA}

Se realizó un estudio correlacional en una población de 1670 adolescentes de secundaria de 11 a 16 años del estado de Puebla, se llevó a cabo un muestreo aleatorio estratificado por turno y grado escolar, por medio de la fórmula de población finita con un nivel de significancia de 95\%, un nivel de error y tamaño de precisión de 
.05, se obtuvo una muestra de 312 participantes. Para la recolección de la información se utilizó una cédula de datos personales para identificar las características sociodemográficas y la Escala de Ansiedad de Hamilton que consta de 14 ítems, en este estudio sólo se consideró del 1 al 13 al eliminar la pregunta que evalua el comportamiento durante la entrevista. Tiene una opción de respuesta tipo Likert que va desde ausente (0) intensidad ligera (1), intensidad media (2), intensidad elevada (3), e intensidad máxima (4). Los ítem del 1 al 6 miden los síntomas psíquicos (humor ansioso, tensión, miedos, in- somnio, funciones intelectuales y humor depresivo) y del 7 al 13 somáticos (musculares, generales, cardiovasculares, respiratorios, gastrointestinales, genitourinarios y del sistema nervioso autónomo). Para el análisis estadístico se obtuvo la sumatoria donde el puntaje mínimo fue 0 y el máximo 52, se establecieron los puntos de corte que la clasifican en ausencia o remisión del trastorno (0 a 5), ansiedad leve (6 a 14) y ansiedad moderada/grave (más de 15). El instrumento mostró un Alpha de Cronbach de .881 que indica consistencia interna aceptable. Ha sido aplicado en diversos estudios en población mexicana y

Tabla 1. Manifestaciones psíquicas de ansiedad en adolescentes

\begin{tabular}{|c|c|c|c|c|c|c|}
\hline \multirow{3}{*}{$\begin{array}{l}\text { Manifestaciones } \\
\text { Síntomas psíquicos }\end{array}$} & \multicolumn{3}{|c|}{ Últimos treinta días } & \multicolumn{3}{|c|}{ Últimos siete días } \\
\hline & \multirow[b]{2}{*}{$\%$} & \multicolumn{2}{|c|}{ IC $95 \%$} & \multirow[b]{2}{*}{$\%$} & \multicolumn{2}{|c|}{ IC $95 \%$} \\
\hline & & LI & LS & & LI & LS \\
\hline \multicolumn{7}{|l|}{ Humor ansioso } \\
\hline Inquietud & 58.8 & 54 & 65 & 54.2 & 48 & 60 \\
\hline Irritabilidad & 38.9 & 33 & 44 & 35.5 & 30 & 41 \\
\hline Aprensión (anticipación temerosa) & 32.9 & 28 & 38 & 26.9 & 20 & 30 \\
\hline \multicolumn{7}{|l|}{ Tensión } \\
\hline Reacciones de sobresalto (enojo) & 51.5 & 46 & 57 & 51.2 & 45 & 57 \\
\hline Fatigabilidad & 41.2 & 36 & 47 & 37.5 & 32 & 43 \\
\hline $\begin{array}{l}\text { Sensaciones de incapacidad para } \\
\text { esperar }\end{array}$ & 39.2 & 34 & 45 & 33.6 & 28 & 39 \\
\hline \multicolumn{7}{|l|}{ Miedos } \\
\hline A quedarse solo & 28.6 & 23 & 34 & 17.9 & 23 & 33 \\
\hline los desconocidos & 35.9 & 30 & 41 & 30.6 & 25 & 36 \\
\hline A la oscuridad & 31.6 & 26 & 37 & 29.6 & 24 & 35 \\
\hline \multicolumn{7}{|l|}{ Insomnio } \\
\hline Sueño interrumpido & 48.2 & 42 & 54 & 41.9 & 36 & 47 \\
\hline $\begin{array}{l}\text { Sueño no satisfactorio, con can- } \\
\text { sancio al despertar }\end{array}$ & 47.5 & 42 & 53 & 41.9 & 44 & 55 \\
\hline $\begin{array}{l}\text { Dificultades de conciliación del } \\
\text { sueño }\end{array}$ & 38.5 & 33 & 44 & 34.6 & 29 & 40 \\
\hline \multicolumn{7}{|l|}{ Funciones intelectuales } \\
\hline Dificultad de concentración & 57.5 & 52 & 63 & 57.8 & 52 & 63 \\
\hline Mala memoria & 47.5 & 42 & 54 & 43.2 & 38 & 49 \\
\hline \multicolumn{7}{|l|}{ Humor depresivo } \\
\hline $\begin{array}{l}\text { Perdida de interés (de las cosas de } \\
\text { la vida) }\end{array}$ & 45.2 & 38 & 49 & 35.2 & 30 & 41 \\
\hline No disfruta del tiempo libre & 39.9 & 34 & 45 & 33.9 & 30 & 41 \\
\hline Insomnio de madrugada & 29.6 & 24 & 35 & 28.6 & 23 & 34 \\
\hline
\end{tabular}

Fuente: Instrumento

Nota: Se incluyen las manifestaciones psíquicas de la ansiedad que se presentaron con mayor frecuencia 
Tabla 2. Manifestaciones somáticas de ansiedad en adolescentes

\begin{tabular}{|c|c|c|c|c|c|c|}
\hline \multirow{3}{*}{$\begin{array}{l}\text { Manifestaciones } \\
\text { Síntomas somáticos }\end{array}$} & \multicolumn{3}{|c|}{ Últimos treinta días } & \multicolumn{3}{|c|}{ Últimos siete días } \\
\hline & \multirow[b]{2}{*}{$\%$} & \multicolumn{2}{|l|}{ IC $95 \%$} & \multirow[b]{2}{*}{$\%$} & \multicolumn{2}{|c|}{ IC $95 \%$} \\
\hline & & LI & LS & & LI & LS \\
\hline \multicolumn{7}{|l|}{ Síntomas somáticos musculares } \\
\hline $\begin{array}{l}\text { Sacudidas clónicas (movimientos rápidos } \\
\text { de pies y manos) }\end{array}$ & 37.5 & 32 & 43 & 33.2 & 28 & 39 \\
\hline Dolores musculares & 36.9 & 31 & 42 & 36.9 & 31 & 42 \\
\hline Sacudidas musculares & 17.6 & 13 & 22 & 14.0 & 10 & 18 \\
\hline \multicolumn{7}{|l|}{ Síntomas somáticos generales } \\
\hline Sensaciones de calor o frío & 52.8 & 47 & 58 & 44.2 & 39 & 50 \\
\hline $\begin{array}{l}\text { Sensaciones parestesias (pinchazos u hor- } \\
\text { migueos en el cuerpo) }\end{array}$ & 40.9 & 35 & 46 & 34.9 & 29 & 40 \\
\hline Visión borrosa & 35.9 & 30 & 41 & 35.5 & 30 & 41 \\
\hline \multicolumn{7}{|l|}{ Síntomas cardiovasculares } \\
\hline Dolor torácico (en el pecho) & 22.6 & 18 & 27 & 18.6 & 14 & 23 \\
\hline Taquicardia (latidos rápidos del corazón) & 17.3 & 13 & 22 & 14.3 & 10 & 18 \\
\hline Palpitaciones (sobresaltos del corazón) & 12.6 & 9 & 16 & 11.6 & 8 & 15 \\
\hline \multicolumn{7}{|l|}{ Síntomas respiratorios } \\
\hline Suspiros & 45.5 & 40 & 51 & 43.2 & 38 & 49 \\
\hline Sensación de ahogo o falta de aire & 31.6 & 26 & 37 & 22.6 & 19 & 28 \\
\hline Disneas (dificultad para respirar) & 18.9 & 14 & 23 & 15.9 & 12 & 20 \\
\hline \multicolumn{7}{|l|}{ Síntomas gastrointestinales } \\
\hline Borborigmos (ruidos intestinales) & 23.3 & 18 & 28 & 16.9 & 13 & 21 \\
\hline Pérdida de peso & 22.3 & 18 & 27 & 19.9 & 15 & 24 \\
\hline Cólicos (espasmos) abdominales & 21.9 & 17 & 27 & 16.6 & 12 & 21 \\
\hline \multicolumn{7}{|l|}{ Síntomas genitourinarios } \\
\hline Micciones frecuentes (orinar) & 17.9 & 14 & 22 & 14.6 & 11 & 19 \\
\hline $\begin{array}{l}\text { Micción imperiosa (muchas ganas de ori- } \\
\text { nar) }\end{array}$ & 12.3 & 9 & 16 & 12.3 & 9 & 16 \\
\hline Amenorrea (falta de periodo menstrual) & 9.6 & 6 & 13 & 8.6 & 5 & 12 \\
\hline \multicolumn{7}{|l|}{ Síntomas del sistema nervioso autónomo } \\
\hline Boca seca & 40.5 & 35 & 46 & 35.5 & 30 & 41 \\
\hline Cefalea por tensión (dolor de cabeza) & 36.9 & 36 & 47 & 36.9 & 31 & 42 \\
\hline Vértigos (mareos) & 25.9 & 24 & 34 & 25.9 & 21 & 31 \\
\hline
\end{tabular}

Nota: Se incluyen las manifestaciones psíquicas de la ansiedad que se presentaron con mayor frecuencia 
es utilizado por la Secretaría de Salud para detectar la ansiedad $^{(7,12,13)}$.

Para conocer manifestaciones psíquicas y somáticas de la ansiedad que se muestran entre los adolescentes se tomaron en cuenta las manifestaciones correspondientes a los síntomas psíquicos y somáticos, se elaboró una encuesta a partir de ellos, en la que se preguntó la presencia (Si) o ausencia (No) durante el último mes y siete días, antes de la recolección de los datos se realizaron pilotajes donde se aplicó en adolescentes de secundaria y estudiantes de licenciatura.

La investigación fue aprobada y autorizada por el comité asignado por la Secretaría de Investigación y Estudios de Posgrado de la Universidad donde procedía el investigador, para llevarlo a cabo se tomó en consideración el Reglamento de la Ley General de Salud en Materia de Investigación para la Salud ${ }^{(15)}$ se respetó la integridad del participante en todo momento y solicitó la firma del consentimiento informado para el padre o tutor y el adolescente. La selección aleatoria del participante sin solicitar el nombre se consideró a partir del número de lista previamente determinado en el programa de Microsoft Excel.

Para analizar los datos se utilizó la estadística descriptiva a través de frecuencias, proporciones y medidas de tendencia central, Prueba de Chi Cuadrada para sexo y edad que se clasificó en dos grupos por medio de rangos y la correlación de Spearman.

\section{RESULTADOS}

Los resultados de este estudio muestran que la mayoría de los estudiantes pertenecieron al primer grado de secundaria (37.9\%), tenían 12 años (33.2\%) y ocho de estudio (34.2\%). En la tabla 1 y 2 se evidenció que los síntomas psíquicos y somáticos más frecuentes en los últimos treinta días y siete, se destaca que, entre los participantes, hubo mayor presencia de síntomas psíquicos donde los porcentajes más elevados que corresponden a su clasificación fueron inquietud, dificultad de concentración, reacciones de sobresalto, sueño interrumpido y no satisfactorio con cansancio al despertar, mala memoria, pérdida de interés de las cosas de la vida y miedo a lo desconocido e irritabilidad. Respecto a los síntomas somáticos con más porcentaje fueron sensaciones de calor y frío, suspiros, sensaciones de parestesias, boca seca, sacudidas clónicas, dolores musculares y cefalea por tensión.

Con relación a la intensidad de los síntomas de ansiedad, se encontró que la mayoría de los adolescentes demostró intensidad ligera y media; donde las manifestaciones psíquicas más prevalentes fueron el humor ansioso, las

Tabla 3. Intensidad de los síntomas de ansiedad en adolescentes

\begin{tabular}{|c|c|c|c|c|c|}
\hline Síntomas de Ansiedad & Ausente & $\begin{array}{c}\text { Intensidad } \\
\text { ligera }\end{array}$ & $\begin{array}{c}\text { Intensidad } \\
\text { media }\end{array}$ & $\begin{array}{c}\text { Intensidad } \\
\text { elevada }\end{array}$ & $\begin{array}{l}\text { Intensidad } \\
\text { máxima }\end{array}$ \\
\hline & $\%$ & $\%$ & $\%$ & $\%$ & $\%$ \\
\hline \multicolumn{6}{|l|}{ Psíquicos } \\
\hline Humor ansioso & 19.9 & 39.9 & 26.6 & 6.6 & 7.0 \\
\hline Tensión & 23.6 & 35.2 & 20.3 & 14.0 & 7.0 \\
\hline Miedos & 31.6 & 27.9 & 19.6 & 12.6 & 8.3 \\
\hline Insomnio & 31.6 & 31.2 & 19.3 & 10.0 & 8.0 \\
\hline Funciones intelectuales & 22.6 & 35.2 & 20.9 & 15.6 & 5.6 \\
\hline Humor depresivo & 44.5 & 24.9 & 13.6 & 9.3 & 7.6 \\
\hline \multicolumn{6}{|l|}{ Somáticos } \\
\hline Musculares & 35.2 & 34.2 & 15.9 & 9.6 & 5.0 \\
\hline Generales & 29.9 & 32.6 & 19.3 & 9.3 & 9.0 \\
\hline Cardiovasculares & 52.8 & 29.2 & 11.3 & 3.7 & 3.0 \\
\hline Respiratorios & 50.5 & 26.9 & 12.6 & 7.3 & 2.7 \\
\hline Gastrointestinales & 58.8 & 27.9 & 8.6 & 3.0 & 1.7 \\
\hline Genitourinarios & 66.4 & 23.3 & 6.6 & 2.0 & 1.7 \\
\hline Del sistema nervioso autónomo & 39.9 & 32.9 & 15.3 & 7.3 & 4.7 \\
\hline
\end{tabular}


Tabla 4. Ansiedad por edad y género en adolescentes de secundaria

\begin{tabular}{|c|c|c|c|c|c|c|c|}
\hline Variables & $f$ & $\%$ & $f$ & $\%$ & $x^{2}$ & gl & $\begin{array}{l}\text { Valor } \\
\text { de } p\end{array}$ \\
\hline Edad & \multicolumn{2}{|c|}{11 a 13} & \multicolumn{2}{|c|}{14 a 16} & & & \\
\hline Remisión del trastorno & 37 & 19.1 & 13 & 12.1 & \multirow{3}{*}{2.584} & \multirow{3}{*}{2} & \multirow{3}{*}{.275} \\
\hline Ansiedad leve & 74 & 38.1 & 47 & 43.9 & & & \\
\hline Ansiedad moderada/grave & 83 & 42.8 & 47 & 43.9 & & & \\
\hline Género & \multicolumn{2}{|c|}{ Hombre } & \multicolumn{2}{|c|}{ Mujer } & & & \\
\hline Remisión del trastorno & 22 & 15.7 & 28 & 17.4 & \multirow{3}{*}{.232} & \multirow{3}{*}{2} & \multirow{3}{*}{.891} \\
\hline Ansiedad Leve & 58 & 41.4 & 63 & 39.1 & & & \\
\hline Ansiedad moderada/grave & 60 & 42.9 & 70 & 43.5 & & & \\
\hline
\end{tabular}

Fuente: Instrumento

funciones intelectuales y la tensión; en cambio las manifestaciones somáticas que más exteriorizaron fueron musculares, generales y del sistema nervioso autónomo (Tabla 3).

No se encontró diferencia estadísticamente significativa entre la intensidad de la ansiedad y los dos grupos de edad $\left(\chi^{2}=2.548, p=.275\right)$ tampoco con el sexo $\left(\chi^{2}=.232, p=.891\right)$. Sin embargo destaca que entre los adolescentes de 11 a 13 años se obtuvo un porcentaje elevado de ansiedad moderada/grave (42.8\%) seguida de ansiedad leve (38.1\%) y ausencia o remisión del trastorno (19.1\%), y que los estudiantes de 14 a 16 años presentaron los mayores porcentajes de ansiedad leve y moderada/grave (43.9\%). En relación con el sexo la ansiedad moderada/grave fue similar en mujeres (43.5\%) y hombres (42.9\%) (Tabla 4). No hubo diferencia estadísticamente significativa entre la ansiedad y la edad $\left(r_{s}=\right.$ $.070, p>.001)$.

\section{DISCUSIÓN Y CONCLUSIONES}

A partir de este estudio se identificaron las manifestaciones psíquicas y somáticas de la ansiedad en adolescentes de secundaria del estado de Puebla, asimismo se determinó la intensidad de la ansiedad y las diferencias por edad y sexo y la relación de la ansiedad con la edad.

Se encontró que prevalecieron los síntomas psíquicos de ansiedad entre los estudiantes de secundaria, sin embargo, también se presentaron síntomas somáticos; entre ellos se puede mencionar la dificultad de concentración, sueño interrumpido no satisfactorio, cefalea por tensión y vértigos lo que coincide con otros reportes de la literatura que señalan a estos síntomas como principales $^{(2,13)}$. Tales resultados sugieren que este grupo etario está manifestando alteraciones cognoscitivas y fi- siológicas que si no se controlan las complicaciones pueden ocasionar problemas durante su desarrollo ${ }^{(1,2)}$, por lo que es necesario que en las instituciones educativas se realicen intervenciones de psicoeducación con la finalidad de generar habilidades de afrontamiento ${ }^{(14)}$.

Por otro lado, la intensidad que demostraron los adolescentes en referencia a los síntomas fue de ligera a media tanto para los psíquicos como somáticos, esto es consistente con los hallazgos anteriores, que pueden reflejarse por la constante presencia durante el último mes y siete días. Cabe señalar que no se evidenciaron estudios que reporten la frecuencia de la intensidad de la ansiedad; sin embargo, los resultados de este estudio confirman lo establecido por la literatura que menciona las dificultades de atención y concentración, problemas de memoria, lentitud del pensamiento, sentimientos de despersonalización y desrealización como principales síntomas durante esta etapa (2), de manera que se hace necesario identificar las causas específicas que provocan la ansiedad para realizar intervenciones de detección y manejo considerando al personal de salud, las autoridades educativas y las familias de los estudiantes.

Con relación a la edad los adolescentes de secundaria de 11 a 16 años mostraron elevados porcentajes de ansiedad (40\%) que van desde leve hasta moderada/grave, esto confirma que la ansiedad se exterioriza durante la adolescencia y que las manifestaciones se mantienen conforme avanza la edad y durante el desarrollo de esta etapa como lo mencionan otras investigaciones $(1,10,11)$.

Además, se evidencia al realizar la comparación por sexo, que existe ansiedad tanto en hombres como en mujeres, situación similar con otro estudio que al igual que este indica no haber diferencia estadísticamente significativa en su población de estudio. A su vez los resultados de esta investigación se asemejan con otras 
fuentes que sugieren que no esta aclarada la presencia de los trastornos de ansiedad por sexo ${ }^{(2,11)}$, esto puede deberse al contexto cultural e incluso escolar donde se desenvuelven los participantes. Respecto a la relación entre la ansiedad y la edad, tampoco se encontró diferencia estadística lo que difiere con Órgiles ${ }^{(9)}$ y colaboradores en el 2012, que indican que a mayor edad mayor nivel de ansiedad. El resultado de este estudio puede deberse a la homogeneidad de los datos al no mostrar una mayor distribución para algún grupo de edad, lo que puede ser por que las manifestaciones clínicas de la ansiedad varían en función de la edad y del desarrollo cognitivo y emocional del individuo ${ }^{(2)}$, de manera que los cambios propios de la adolescencia y la presencia de los síntomas que estos tienen pueden influir en este resultado.

Se concluye que los adolescentes están manifestando y manteniendo síntomas psíquicos y somáticos de ansiedad desde edades tempranas, lo que puede ser alarmante ya que la presencia de estos puede traer consigo problemas de disfunción y desadaptación en la vida del adolescente, que al mismo tiempo puedan desencadenar problemas en la salud física y mental que los lleve a realizar conductas de riesgo. Se sugiere que se continúen realizando estudios que aborden la variable ansiedad y se evalúen los síntomas con medidas de frecuencia de hasta seis meses con la intención de poder establecer un mejor diagnóstico sobre esta problemática.

Por lo tanto la finalidad de este estudio fue identificar la problemática de los síntomas de la ansiedad para enfocar intervenciones al cuidado del adolescente y contribuir a mejorar la calidad de vida. La limitación de este estudio radicó en las pocas investigaciones encontradas y las diferencias en cuanto a su medición. Sin embargo, los resultados contribuyen en la generación del conocimiento para que a partir de ello se generen intervenciones de enfermería sustentadas por evidencia científica que ayuden a ofrecer un mejor cuidado hacia esta población.

\section{REFERENCIAS BIBLIOGRÁFICAS}

1.Cárdenas EM, Feria M, Palacios L, De la Peña F. Guía Clínica para los trastornos de Ansiedad en Niños y Adolescentes. Instituto Nacional de Psiquiatría. (Serie: Guías Clínicas para la Atención de Trastornos Mentales). 2010. México. [Citado 18 febrero 2018]; Disponible a partir de: http://inprf-cd.gob.mx/guiasclinicas/trastornos_de_ansiedad.pdf

2.Mardomingo MJ. Trastornos de ansiedad en el adolescente. Pediatr Integral. 2005 [Citado 18 febrero 2018]; 9(2): 125-134. Disponible a partir de: https://faros.hsjdbcn.org/adjuntos/336.1-Trastornos_ansiedad_adolescente(1).pdf
3.Gorosabel J. Estudio de los síntomas somáticos asociados a los trastornos depresivos y de ansiedad en una población de atención primaria. [Tesis doctoral]. España: Universidad Autónoma de Madrid; 2013 [Citado 23 marzo 2018]; Disponible a partir de: https://repositorio.uam. es/bitstream/handle/10486/14125/66216_gorosabel\%20 rebolleda\%20jesus.pdf? sequence $=1$

4.Asociación Americana de Psiquiatría [APA]. Guía de consulta de los criterios diagnósticos de DSM 5. 2013. Arlington, V.A., Asociación Americana de Psiquiatría. [Citado 24 marzo 2018]; Disponible a partir de: http://www. psicoaragon.es/wp-content/uploads/2017/06/DSM-5. pdf

5.Bendini S. ¿Qué es la ansiedad? Salud Mental. 2013; 14(2): 113-21.

6.Martínez M. M.C., García F. J. M., Cándido J.I. Relaciones entre ansiedad escolar, ansiedad rasgo, ansiedad estado y depresión en una muestra de adolescentes españoles. International Journal of Psychology and Psychological Therapy. 2013 [Citado 9 abril 2018]; 1(13): 47-64. Disponible a partir de: https://www.ijpsy.com/volumen13/ num $1 / 346 /$ relaciones-entre-ansiedad-escolar-ansiedad-ES.pdf

7.Secretaría de Salud. Guía de Práctica Clínica Diagnóstico y Tratamiento de los Trastornos de Ansiedad en el Adulto. 2010 [Citado 9 abril 2018]; México. Disponible a partir de http://www.cenetec.salud.gob.mx/descargas/gpc/CatalogoMaestro/392_IMSS_10_Ansiedad/EyR_IMSS_392_10. pdf

8.Shorter E. A historical dictionary of psychiatry. Oxford University Press. 2005. New York. 338 p.

9.Orgilés M, Méndez X, Espada JP, Carballo JL, Piqueras JA. Síntomas de trastornos de ansiedad en niños y adolescentes: diferencias en función de la edad y el sexo en una muestra comunitaria. Rev Psiquiatr Salud Ment (Barc.). 2012 [Citado 8 mayo 2018]; (2):115-20. Disponible a partir de: file:///C:/Users/150005990/Downloads/ S1888989112000237_S300_es.pdf

10.Benjet C, Borges G, Medina-Mora ME, Méndez E, Fleiz C, Rojas E, Cruz A. Diferencias de sexo en la prevalencia y severidad de trastornos psiquiátricos en adolescentes de la Ciudad de México. Salud Mental. 2009 [Citado 9 mayo 2018]; 32(2): 155-63. Disponible a partir de: http://www. redalyc.org/pdf/582/58212275008.pdf

11.Jiménez-López LM, Jurado CS. Ansiedad social consumo riesgoso de alcohol en adolescentes mexicanos. Journal of Behavior, Healt \& Social Issues. 2014 [Citado 14 mayo 2018]; 1(6): 37-50. Disponible a partir de: http:// www.scielo.org.mx/pdf/jbhsi/v6n1/v6n1a3.pdf

12.Ramírez-Osorio P, Hernández-Mendoza E. Resiliencia 
familiar, depresión y ansiedad en adolescentes en situación de pobreza. Rev Enferm Inst Mex Seguro Soc. 2012 [Citado 25 mayo 2018]; 20(2): 63-70. Disponible a partir de: http://www.medigraphic.com/pdfs/enfermeriaimss/ eim-2012/eim122b.pdf

13.Secretaría de Salud. Intervenciones de enfermería para la detección oportuna y control de los trastornos de ansiedad en niños y adolescentes. Resumen de Evidencias y Recomendaciones: Guía de Práctica clínica de enfermería. México: CENETEC; 2017. [Citado 26 mayo 2018]. Disponible a partir de http://www.cenetec.salud.gob.mx/ contenidos/gpc/catalogoMaestroGPC.html\#

14.Fuentes CRM, Simón SMJ, Garrido AM, Serrano PMD, Larrañaga RME, Yubero JS. Síntomas psicosomáticos como expresión del deterioro de la calidad de vida relacionada con la salud en adolescentes. Aten Primaria. 2017 [Citado 26 mayo 2018]; 1-7. Disponible a partir de: https://doi.org/10.1016/j.aprim.2017.06.009

15.Secretaría de Salud. Ley general de salud en materia de investigación. México. SS; 2015 [Citado 26 mayo 2018]. Disponible a partir de: www.salud.gob.mx/unidades/cdi/nom/compi/rlgsmis.ht 